\title{
THE GENERAL INVARIANT THEORY OF IRREGULAR ANALYTIC ARCS OR ELEMENTS
}

\author{
BY \\ EDWARD KASNER AND JOHN DECICCO
}

Introduction. In this paper, we shall begin the study of the invariant theory of the most general irregular analytic arc in the geometry based on the infinite group $G$ of arbitrary regular point transformations. Our results are valid for the group of real point transformations of the real plane; or for the group of complex point transformations of the complex plane. Kasner has developed the corresponding theory in the conformal geometry of the complex plane $\left(^{1}\right)$. The present paper opens up a new aspect of restricted topology.

Our subject is then the equivalence theory of a single arc or curve. When can one analytic arc be converted into another analytic arc by an arbitrary regular point transformation of the plane? It is apparently implied, in the current literature, that there is no problem here. For any curve (it is implied) can be converted into any other, in particular, into the $x$-axis. But this is based on the assumption that the arcs are regular. If we give up this assumption, we have actual problems which certainly seem worthy of treatment. Our subject is therefore the invariant theory of a general irregular analytic arc under the group $G$ of arbitrary regular point transformations.

More exactly, the configuration we shall discuss is not simply an analytic arc but rather that arc together with a specific point of the arc. This compound configuration we shall term an analytic element. It consists of a pointthe base point which shall be taken as the origin throughout this paper-and an analytic arc through the point. It may be described also as a differential element of infinite order $\left({ }^{2}\right)$.

The most general analytic element, if the given point $o$ is taken as origin, is represented by writing $x$ and $y$ as integral power series in a parameter $t$ without the constant terms. If the parameter $t$ is eliminated, then $y$ is found as a series in $x$ which may proceed according to integral or fractional powers

Presented to the Society, January 1, 1941, under the title The classification of analytic arcs or elements under the group of arbitrary point transformations; received by the editors January 2, 1941.

(1) Kasner, Conformal classification of analytic arcs or elements; Poincarế's local problem of conformal geometry, these Transactions, vol. 16 (1915), pp. 333-349.

(2) Kasner has introduced elsewhere the concept of divergent differential element of infinite order. This corresponds to a divergent power series and may be represented by a nonanalytic arc having specified values for all the successive derivatives. Thus to every power series corresponds a geometric entity which may be real or imaginary, regular or irregular, convergent or divergent. This entity is the most general differential element. If it is convergent, we call it an analytic element (regular or irregular), or more loosely, an analytic arc or a curve. 
of $x$. If fractional exponents enter and cannot be avoided by interchanging $x$ and $y$ (this will then necessarily be the case for any choice of rectangular axes), we shall call the element irregular $\left(^{3}\right)$; otherwise the element is regular.

Our new problem is to classify with respect to the group $G$ of arbitrary regular point transformations all irregular analytic elements.

It is obvious that all regular elements are equivalent under the group $G$ of arbitrary point transformations. That is, all complex regular elements (both real and imaginary) are equivalent under the group $G$ of complex point transformations; whereas all real regular elements are equivalent under the group $G$ of all real point transformations. Any regular element may be reduced to the canonical form $y=0$ (the $x$-axis together with the origin as base point).

But for irregular elements, the results are quite complicated. (See the table on page 235.) It is clear, for example, that the cuspidal element $y=x^{3 / 2}$ cannot be converted into the regular element $y=0$, nor into the irregular element $y=x^{4 / 3}$. For these curves differ qualitatively in the nature of the singular point at the origin. However, suppose the two proposed elements have the same kind of irregularity (in a sense to be later defined, depending on agreement of certain exponents-certain arithmetic invariants); will they necessarily be equivalent? If not, certain combinations of the coefficients will be invariant, that is, there will be absolute differential invariants. For example, we find that every differential element of the form

$$
y=x^{3 / 2}+c_{4} x^{4 / 2}+c_{5} x^{5 / 2}+\cdots,
$$

can be (formally) reduced to $y=x^{3 / 2}$. On the other hand, not every element of the form

$$
y=x^{9 / 4}+c_{10} x^{10 / 4}+c_{11} x^{11 / 4}+\cdots,
$$

can be reduced to $y=x^{9 / 4}$. Hence in the first type there are no invariants; in the second type there exists an invariant.

In general, irregular elements have absolute differential invariants; certain exceptions arise, namely, those in which the corresponding series in $x$ proceeds according to powers of the square root, and the cube root, and three particular types of series in $x$ which proceed according to powers of the fourth root. All the other cases possess absolute differential invariants.

Statement of results. We shall throughout this paper assume that our group $G$ of arbitrary regular point transformations leaves invariant the fixed point $o$ (the origin) of our analytic element and that it is regular in the neighborhood of $o$. Thus our group $G$ is

${ }^{(3)}$ This is related to the concept of cycle used in the theory of algebraic curves, and to the more general theory of algebroid arcs. Topologic invariants of algebroid arcs are studied in papers by Brauner, Kähler, and Zariski; for references see the latter's paper, American Journal of Mathematics, vol. 54 (1932), pp. 453-465. 


$$
\begin{aligned}
X= & \left(\alpha_{01} x+\alpha_{02} x^{2}+\cdots\right)+y\left(\alpha_{10}+\alpha_{11} x+\cdots\right) \\
& +y^{2}\left(\alpha_{20}+\alpha_{21} x+\cdots\right)+\cdots, \\
Y= & \left(\beta_{01} x+\beta_{02} x^{2}+\cdots\right)+y\left(\beta_{10}+\beta_{11} x+\cdots\right) \\
& +y^{2}\left(\beta_{20}+\beta_{21} x+\cdots\right)+\cdots,
\end{aligned}
$$

where the jacobian $J=\alpha_{01} \beta_{10}-\alpha_{10} \beta_{01} \neq 0$. We therefore shall study the invariant theory of an analytic element (with the origin as base point) with respect to the group $G$ as given by these equations.

Any analytic element with origin as base point may be defined by setting $x$ and $y$ equal to two power series in a parameter $t$ without constant terms. Let $p \geqq 1$ be the minimum of the two exponents of the two leading terms of the two power series in $t$. By interchanging the coordinates $x$ and $y$ appropriately, we can always arrange that the exponent of the leading term of the power series defining the abscissa $x$ shall be our number $p$. Therefore any analytic element may be written in the form

$$
y=c_{p} x^{p / p}+c_{2 p} x^{2 p / p}+\cdots+c_{r p} x^{r p / p}+c_{q} x^{q / p}+c_{q+1} x^{(q+1) / p}+\cdots,
$$

where $q$ is the first $q$ th power of the $p$ th root of $x$ which is not a multiple of $p$. That is, the integer $q=r p+s$ is such that the integers $r$ and $s$ satisfy the inequalities $r \geqq 1$ and $0<s<p$.

If $c_{q} \neq 0$, our element is said to be irregular. Otherwise our element is said to be regular. Thus an element is regular if $p=1$ (and hence no such term $c_{q} x^{q / p}$ can appear in our power series); or if $p \geqq 2$ and if $c_{q}=0$ (the coefficient of any actual fractional power of $x$ is zero).

For an irregular element the integer $p \geqq 2$ is called the index and the integer $q=r p+s, r \geqq 1,0<s<p$, is termed the rank. All irregular elements obtained by taking arbitrary values of the coefficients, but fixing the values of both $p$ and $q$, we shall define as forming the single species $(p, q)$. It is proved that the index $p$ and the rank $q$ are arithmetic invariants. Our main result follows:

Absolute differential invariants, that is, functions of the coefficients unaltered by the group $G$ of arbitrary point transformations, exist for all irregular species $(p, q)$ except in the cases $(4,5),(4,6),(4,7),(3, q)$, and $(2, q)$. The species $(4,5)$ may be divided into two distinct sets, and the species $(4,7)$ may be separated into three distinct sets; the elements of any one of these sets are all equivalent to each other. The species $(4,6)$ and $(3, q)$ may be separated into a denumerably infinite number of such distinct sets. All the elements of the species $(2, q)$ are equivalent to each other-the canonical form is $y=x^{q / 2}$. Finally all the regular elements are obviously transformable into each other, the standard form being $y=0$.

The species $(p, q=r p+s)$ for which $r \geqq 2$ and $0<s<p-2$, or for which $r=1$ and $2<s<p-2$, possesses an absolute differential invariant of order 
$q+2$ (and none of lower order). This invariant involves only the coefficients $c_{q}, c_{q+1}$ and $c_{a+2}$.

The species $(p, q=r p+s)$ for which $r \geqq 2$ and $1<s=p-2$, or for which $r=1$ and $3<s=p-2$, possesses an absolute differential invariant of order $q+3$. This involves only the coefficients $c_{q}, c_{q+1}$, and $c_{q+3}$.

The species $(p, q=r p+s)$ for which $r \geqq 2$ and $2<s=p-1$, or for which $r=1$ and $3<s=p-1$, possesses an absolute differential invariant of order $q+3$. This involves only the coefficients $c_{q}, c_{q+2}$, and $c_{q+3}$.

The species $(p, q=p+1)$ for which $p>4$ possesses an absolute differential invariant of order $q+3=p+4$. This contains only the coefficients $c_{p+1}, c_{p+2}$, $c_{p+3}$, and $c_{p+4}$.

The species $(p, q=p+2)$ for which $p>5$ possesses an absolute differential invariant of order $q+3=p+5$. This contains only the coefficients $c_{p+2}, c_{p+3}$, $c_{p+4}$, and $c_{p+5}$.

The species $(5,7)$ possesses an absolute differential invariant of order 11. This contains only the coefficients $c_{7}, c_{8}, c_{9}$, and $c_{11}$.

Finally the species $(5,8)$ possesses an absolute differential invariant of order 12 . This involves only the coefficients $c_{8}, c_{9}, c_{11}$, and $c_{12}$.

The following table exhibits some of the results in detail.

\begin{tabular}{r|rrrrrrrrrrrrrrrr}
\hline & 3 & 4 & 5 & 6 & 7 & 8 & 9 & 10 & 11 & 12 & 13 & 14 & 15 & 16 & 17 & 18 \\
\hline 2 & $*$ & & $*$ & & $*$ & & $*$ & & $*$ & & $*$ & & $*$ & & $*$ & \\
3 & & $*$ & $*$ & & $\dagger$ & $\dagger$ & & $\dagger$ & $\dagger$ & & $\dagger$ & $\dagger$ & & $\dagger$ & $\dagger$ & \\
4 & & & $\dagger$ & $\ddagger$ & $\dagger$ & & 11 & 13 & 14 & & 15 & 17 & 18 & & 19 & 21 \\
5 & & & & 9 & 11 & 12 & 12 & & 13 & 14 & 16 & 17 & & 18 & 19 & 21 \\
6 & & & & & 10 & 11 & 11 & 13 & 14 & & 15 & 16 & 17 & 19 & 20 & \\
7 & & & & & & 11 & 12 & 12 & 13 & 15 & 16 & & 17 & 18 & 19 & 20 \\
8 & & & & & & & 12 & 13 & 13 & 14 & 15 & 17 & 18 & & 19 & 20 \\
9 & & & & & & & & 13 & 14 & 14 & 15 & 16 & 17 & 19 & 20 & \\
10 & & & & & & & & & 14 & 15 & 15 & 16 & 17 & 18 & 19 & 21 \\
\hline
\end{tabular}

Here the species is determined by the value of $p$ in the left column and the value of $q$ in the top row. The blank spaces denote the fact that there are no such species. In the body of the table we find the order of the absolute differential invariants discussed above. The asterisk indicates that the corresponding species has no absolute invariant and further that all members of that species are equivalent under the group $G$ of arbitrary point transformations. The dagger indicates that there are no absolute differential invariants but that the members are not all equivalent: there exists a certain relative differential invariant, hence there is a division of such a species into a finite number of subspecies, all distinct with respect to the group $G$ of arbitrary 
point transformations. On the other hand, the double dagger indicates that there exists a certain arithmetic invariant and hence the species $(4,6)$ can be divided into an infinite number of subspecies.

1. Discussion of the regular elements. Every regular element: $y=c_{1} x+c_{2} x^{2}+\cdots$, is carried by the point transformation: $X=x, \quad Y=y$ $-\left(c_{1} x+c_{2} x^{2}+\cdots\right)$, into the $x$-axis: $y=0$. Hence

THEOREM 1. All regular analytic elements are equivalent under the group $G$ of arbitrary point transformations. The normal form of a regular analytic element is $y=0$ (the $x$-axis together with the origin as base point).

Our purpose in stating this elementary result is to give a complete classification of all elements both regular and irregular. Henceforth we only have to confine our attention to irregular elements. In the next section, we shall discuss the index $p$ and the rank $q$ of any irregular element.

2. The invariance of the index $p$ and the rank $q$. First let us note that by the point transformation

$$
X=x, \quad Y=y-\left(c_{p} x+c_{2 p} x^{2}+c_{3 p} x^{3}+\cdots+c_{r p} x^{r}\right),
$$

our irregular element (2) is carried into the irregular analytic element

$$
y=c_{q} x^{q / p}+c_{q+1} x^{(q+1) / p}+\cdots, \quad c_{q} \neq 0,
$$

where the coefficients $c_{q}, c_{q+1}, c_{q+2}, \cdots$ of this element are identical with the corresponding ones of our irregular element (2). Thus the transformation (3) eliminates the first $r$ integral powers of $x$.

Applying any transformation of our group $G$ to this element (4), we find as the parametric equations of our new element

$$
\begin{aligned}
& X=\alpha_{01} t^{p}+\alpha_{02} t^{2 p}+\cdots+\alpha_{0 r} t^{r p}+\alpha_{10} c_{q} t^{q}+\cdots, \\
& Y=\beta_{01} t^{p}+\beta_{02} t^{2 p}+\cdots+\beta_{0 r} t^{r p}+\beta_{10} c_{q} t^{q}+\cdots
\end{aligned}
$$

Since the jacobian of the transformation is not zero, at least one of the quantities $\alpha_{01}$ or $\beta_{01}$ is not zero. Hence the number $p$ is the minimum exponent in the two power series defining our new element and therefore must be its index. Thus the group $G$ preserves the index $p$.

By interchanging the coordinates $X$ and $Y$ appropriately, we can always arrange so that $\alpha_{01} \neq 0$. Upon setting $X=T^{p}$, the first of the preceding equations defines $t$ as an integral power series in $T$. This power series must be of the form

$$
\begin{aligned}
t= & A_{1} T+A_{p+1} T^{p+1}+A_{2 p+1} T^{2 p+1}+\cdots \\
& +A_{r p-p+1} T^{r-p+1}+A_{q-p+1} T^{q-p+1}+\cdots,
\end{aligned}
$$

where the exponents after the last written term increase by one (as far as we know). 
Substituting this into the first of equations (5), we obtain

$$
\begin{array}{r}
\alpha_{01} A_{1}^{p}=1, \\
p \alpha_{01} A_{1}^{p-1} A_{p+1}+\alpha_{02} A_{1}^{2 p}=0, \\
p \alpha_{01} A_{1}^{p-1} A_{2 p+1}+\cdots+\alpha_{03} A_{1}^{3 p}=0, \\
p \alpha_{01} A_{1}^{p-1} A_{r p-p+1}+\cdots+\alpha_{0 r} A_{1}^{r p}=0, \\
p \alpha_{01} A_{1}^{p-1} A_{q-p+1}+\alpha_{10} c_{q} A_{1}^{q}=0,
\end{array}
$$

This system of equations determines the $A$ 's in terms of the $\alpha$ 's. We note that $A_{1} \neq 0$. It is also important to note that the last written equation contains only two terms.

If $\left(\alpha_{01}\right)^{1 / p}$ denotes one and only one pth root of $\alpha_{01}$, then it is easily seen that these equations determine a one-to-one correspondence between $\left(\alpha_{01}, \alpha_{02}, \cdots, \alpha_{0 r}\right)$ and $\left(A_{1}, A_{p+1}, A_{2 p+1}, \cdots, A_{r p-p+1}\right)$. Hence we may replace the preceding set of unknown $\alpha$ 's by the latter set of unknown $A$ 's.

Also let us note the following result

$$
J=\alpha_{01} \beta_{10}-\alpha_{10} \beta_{01}=\frac{1}{c_{q} A_{1}^{q+1}}\left(\beta_{10} A_{1}^{q-p+1}{ }^{q}+p \beta_{01} A_{q-p+1}\right) \neq 0 .
$$

This is needed for the completion of our argument.

Now substituting (6) into the second of equations (5), our new element (5) may be written in the form

$$
\begin{aligned}
Y= & \beta_{01} A_{1}^{p} X^{p / p}+\left(\beta_{02} A_{1}^{2 p}+p \beta_{01} A_{1}^{p-1} A_{2 p+1}\right) X^{2 p / p}+\left(\beta_{03} A_{1}^{3 p}+\cdots\right) X^{3 p / p} \\
& +\cdots+\left(\beta_{0 r} A_{1}^{r p}+\cdots\right) X^{r p / p} \\
& +A_{1}^{p-1}\left[\beta_{10} A_{1}^{q-p+1} c_{q}+p \beta_{01} A_{q-p+1}\right] X^{q / p}+\cdots
\end{aligned}
$$

This equation shows that the first power of the pth root of $X$ which is not a multiple of $p$ is the integer $q$. The coefficient of this power is not zero because of (8). Hence the rank $q$ is also preserved.

TheOREM 2. The index $p$ and the rank $q$ of an irregular element are both arithmetic invariants under the group $G$ of arbitrary point transformations.

This theorem justifies our definition of the species $(p, q)$. That is, under the group of arbitrary point transformations any species $(p, q)$ of irregular elements is carried into itself.

By the above equation, it is found that the subgroup $G^{\prime}$ of the group $G$ which carries the element (4) into one of the same form is 
(10) $G^{\prime}$

$$
\begin{aligned}
& X=\left(\alpha_{01} x+\alpha_{02} x^{2}+\cdots\right)+y\left(\alpha_{10}+\alpha_{11} x+\cdots\right)+\cdots, \\
& Y=\left(\beta_{0, r+1} x^{r+1}+\beta_{0, r+2} x^{r+2}+\cdots\right)+y\left(\beta_{10}+\beta_{11} x+\cdots\right)+\cdots,
\end{aligned}
$$

where

$$
\alpha_{01} \beta_{10} \neq 0 .
$$

Since our group $G$ may be factored into the product of the group (3) by the group $G^{\prime}$, and since the group (3) preserves the coefficients $c_{q}, c_{q+1}, \cdots$, it is necessary merely to study the invariant theory of the group $G^{\prime}$.

In the following, we shall find the different invariants of lowest order of our irregular elements. For this purpose, we shall find it convenient to classify our elements according to the types of invariants which arise. See $\S \S 3$ to 14 which follow. Some of the twelve classes are simple and some are complicated. The invariants found vary greatly in structure it will be observed.

3 . The discussion of the species $(p, q=r p+s)$ for which $r \geqq 2$ and $0<s<p-2$, or for which $r=1$ and $2<s<p-2$. Substituting (6) into the second of equations (10), we find because of our inequalities the following transformation formulas between $\left(c_{q}, c_{q+1}, c_{q+2}\right)$ and $\left(C_{q}, C_{q+1}, C_{q+2}\right)$ :

$$
C_{q}=\beta_{10} A_{1}^{q} c_{q}, \quad C_{q+1}=\beta_{10} A_{1}^{q+1} c_{q+1}, \quad C_{q+2}=\beta_{10} A_{1}^{q+2} c_{q+2} .
$$

These equations immediately yield the following result.

THEOREM 3. The species $(p, q=r p+s)$ for which $r \geqq 2$ and $0<s<p-2$, or for which $r=1$ and $2<s<p-2$ possesses the absolute differential invariant of lowest order

$$
\frac{c_{q} c_{q+2}}{c_{q+1}^{2}}
$$

The order of our invariant is $q+2$, its weight is $2 q+2$, and its degree is 2 .

4. The discussion of the species $(p, q=r p+s)$ for which $r \geqq 2$ and $1<s=p-2$, or for which $r=1$ and $3<s=p-2$. Again because of our inequalities, we obtain the following transformation formulas between $\left(c_{q}, c_{q+1}, c_{q+3}\right)$ and $\left(C_{q}, C_{q+1}, C_{q+3}\right)$ :

$$
C_{q}=\beta_{10} A_{1}^{q} c_{q}, \quad C_{q+1}=\beta_{10} A_{1}^{q+1} c_{q+1}, \quad C_{q+3}=\beta_{10} A_{1}^{q+3} c_{q+3} .
$$

Therefore

THEOREM 4. This species possesses the absolute invariant

$$
\frac{c_{q}^{2} c_{q+3}}{c_{q+1}^{3}}
$$

The order is $q+3$, weight is $3 q+3$, and degree is 3 . 
5. The discussion of the species $(p, q=r p+s)$ for which $r \geqq 2$ and $2<s=p-1$, or for which $r=1$ and $3<s=p-1$. Because of these inequalities, the following transformation formulas between $\left(c_{q}, c_{q+2}, c_{q+3}\right)$ and $\left(C_{q}, C_{q+2}\right.$, $C_{q+3}$ ) are obtained:

$$
C_{q}=\beta_{10} A_{1}^{q} c_{q}, \quad C_{q+2}=\beta_{10} A_{1}^{q+2} c_{q+2}, \quad C_{q+3}=\beta_{10} A_{1}^{q+3} c_{q+3} .
$$

THEOREM 5. This species possesses the absolute invariant

$$
\frac{c_{q} c_{q+3}^{2}}{c_{q+2}^{3}} .
$$

The order is $q+3$, weight is $3 q+6$, and degree is 3 .

6. Discussion of the species $(p, q=p+1)$ for which $p>4$. For the case $q=p+1$, we find that the parametric equations (5) for our transformed element assume the form

$$
\begin{aligned}
& X=\alpha_{01} t^{p}+\alpha_{10}\left(c_{p+1} t^{p+1}+c_{p+2} t^{p+2}+c_{p+3} t^{p+3}+\cdots\right)+\cdots \\
& Y=\beta_{10}\left(c_{p+1} t^{p+1}+c_{p+2} t^{p+2}+c_{p+3} t^{p+3}+c_{p+4} t^{p+4}+\cdots\right)+\cdots
\end{aligned}
$$

Let $X=T^{p}$. The first of the preceding equations defines $t$ as an integral power series in $T$. This power series must be of the form

$$
t=A_{1} T+A_{2} T^{2}+A_{3} T^{3}+\cdots, \quad A_{1} \neq 0 .
$$

Substituting this value of $t$ into the first of the preceding equations, we obtain the following system of equations:

$$
\begin{aligned}
& \alpha_{01} A_{1}^{p}=1, \\
& \alpha_{01}\left[p A_{1}^{p-1} A_{3}+\frac{p}{2}(p-1) A_{1}^{p-2} A_{2}^{2}\right] \\
& +\alpha_{10}^{p-1} A_{2}+\alpha_{10} A_{1}^{p+1} c_{p+1}=0 \\
& \alpha_{01}\left[p A_{1}^{p-1} A_{4}+p(p-1) A_{1}^{p} A_{2} c_{p+1}+A_{1}^{p+2} c_{p+2}\right]=0 \\
& \left.+\alpha_{10}^{p-2} A_{2} A_{3}+\frac{p}{6}(p-1)(p-2) A_{1}^{p-3} A_{2}^{3}\right] \\
& \left.+(p+1) A_{1}^{p} A_{3}+\frac{p}{2}(p+1) A_{1}^{p-1} A_{2}^{2}\right\} c_{p+1} \\
& \left.+(p+2) A_{1}^{p+1} A_{2} c_{p+2}+A_{1}^{p+3} c_{p+3}\right]=0
\end{aligned}
$$

Now if $c_{p+1} \neq 0$, we find that $A_{1}$ and $A_{2}$ are arbitrary, but $A_{3}$ and $A_{4}$ are dependent, being given by 
(20)

$$
\begin{aligned}
& A_{3}=\frac{1}{2}(p+3) \frac{A_{2}^{2}}{A_{1}}+A_{1} A_{2} \frac{c_{p+2}}{c_{p+1}} \\
& A_{4}=\frac{1}{3}(p+2)(p+4) \frac{A_{2}^{3}}{A_{1}^{2}}+(p+4) A_{2}^{2} \frac{c_{p+2}}{c_{p+1}}+A_{1}^{2} A_{2} \frac{c_{p+3}}{c_{p+1}}
\end{aligned}
$$

Substituting (18) into the second of equations (17), we discover the following transformation formulas between $\left(c_{p+1}, c_{p+2}, c_{p+3}, c_{p+4}\right)$ and $\left(C_{p+1}, C_{p+2}, C_{p+3}, C_{p+4}\right)$ :

$$
\begin{aligned}
& C_{p+1}=\beta_{10} A_{1}^{p+1} c_{r+1}, C_{p+2}=\beta_{10}\left\{A_{1}^{p+2} c_{p+2}+(p+1) A_{1}^{p} A_{2} c_{p+1}\right\}, \\
& C_{p+3}=\beta_{10}\left\{A_{1}^{p+3} c_{p+3}+(p+2) A_{1}^{p+1} A_{2} c_{p+2}\right.\left.+\left[(p+1) A_{1}^{p} A_{3}+\frac{p}{2}(p+1) A_{1}^{p-1} A_{2}^{2}\right] c_{p+1}\right\}, \\
& C_{p+4}=\beta_{10}\left\{A_{1}^{p+4} c_{p+4}+\right.(p+3) A_{1}^{p+2} A_{2} c_{p+3} \\
&+ {\left[(p+2) A_{1}^{p+2} A_{3}+\frac{1}{2}(p+1)(p+2) A_{1}^{p} A_{2}^{2}\right] c_{p+2} } \\
&+ {\left[(p+1) A_{1}^{p} A_{4}+p(p+1) A_{1}^{p-1} A_{2} A_{3}\right.} \\
&\left.\left.+\frac{p}{6}(p-1)(p+1) A_{1}^{p-2} A_{2}^{3}\right] c_{p+1}\right\} .
\end{aligned}
$$

Eliminating $\beta_{10}, A_{1}, A_{2}, A_{3}$, and $A_{4}$ from these and the preceding equations, we find

THEOREM 6. The species $(p, q=p+1)$ for which $p>4$ possesses the absolute invariant

$$
\frac{\left[(p+1)^{2} c_{p+1}^{2} c_{p+4}-2(p+1)(p+2) c_{p+1} c_{p+2} c_{p+3}+\frac{1}{3}(p+2)(3 p+5) c_{p+2}^{3}\right]^{2}}{\left[(p+1) c_{p+1} c_{p+3}-\frac{1}{2}(2 p+3) c_{p+2}^{2}\right]^{3}} .
$$

The order is $q+3=p+4$; weight is $3 q+3=3 p+6$, and degree is 6 .

7. Discussion of the species $(p, q=p+2)$ for which $p>5$. The calculations for our transformed element will be given by the equations of the preceding section if we assume $c_{p+1}=0$ but $c_{p+2} \neq 0$.

Under this restriction we find that $A_{1} \neq 0$ and $A_{3}$ are arbitrary but $A_{2}$ and $A_{4}$ are dependent, being given by 


$$
A_{2}=0, \quad A_{4}=A_{1} A_{3} \frac{c_{p+3}}{c_{p+2}}
$$

The transformation formulas between $\left(c_{p+2}, c_{p+3}, c_{p+4}, c_{p+5}\right)$ and $\left(C_{p+2}\right.$, $\left.C_{p+3}, C_{p+4}, C_{p+5}\right)$ are

$$
\begin{aligned}
& C_{p+2}=\beta_{10} A_{1}^{p+2} c_{p+2}, \quad C_{p+3}=\beta_{10} A_{1}^{p+3} c_{p+3}, \\
& C_{p+4}=\beta_{10}\left[A_{1}^{p+4} c_{p+4}+(p+2) A_{1}^{p+3} A_{3} c_{p+2}\right], \\
& C_{p+5}=\beta_{10}\left[A_{1}^{p+5} c_{p+5}+(p+3) A_{1}^{p+2} A_{3} c_{p+3}+(p+2) A_{1}^{p+1} A_{4} c_{p+2}\right] .
\end{aligned}
$$
find

Performing the elimination of $\beta_{10}, A_{1}, A_{3}$, and $A_{4}$ from these equations, we

THEOREM 7. The species $(p, q=p+2)$ for which $p>5$ possesses the absolute invariant

$$
\frac{c_{p+2}\left[(p+2) c_{p+2} c_{p+5}-(2 p+5) c_{p+3} c_{p+4}\right]}{c_{p+3}^{3}} .
$$

The order is $q+3=p+5$, weight is $3 q+3=3 p+9$, and degree is 3 .

8. Discussion of the species $(5,7)$. For the species $(p=5, q=7)$, it is found that the parametric equations (5) of the transformed element assume the form

$$
\begin{aligned}
& X=\alpha_{01} t^{5}+\alpha_{10}\left(c_{7} t^{7}+c_{8} t^{8}+c_{9} t^{9}+\cdots\right)+\cdots, \\
& Y=\beta_{10}\left(c_{7} t^{7}+c_{8} t^{8}+c_{9} t^{9}\right)+\left(\beta_{02}+\beta_{10} c_{10}\right) t^{10}+\beta_{10} c_{11} t^{11}+\cdots .
\end{aligned}
$$

Let $X=T^{5}$. The first of the preceding equations defines $t$ as an integral power series in $T$. This power series must be of the form

$$
t=A_{1} T+A_{3} T^{3}+A_{4} T^{4}+A_{5} T^{5}+\cdots, \quad A_{1} \neq 0 .
$$

Replacing $t$ by this in the first of equations (26), we find

$$
\begin{aligned}
& \alpha_{01} A_{1}^{\mathfrak{5}}=1, \quad 5 \alpha_{01} A_{1}^{4} A_{3}+\alpha_{10} A_{1}^{7} C_{7}=0, \\
& 5 \alpha_{01} A_{1}^{4} A_{4}+\alpha_{10} A_{1}^{8} c_{8}=0 \text {, } \\
& \alpha_{01}\left(5 A_{1}^{4} A_{5}+10 A_{1}^{3} A_{3}^{2}\right)+\alpha_{10}\left(7 A_{1}^{6} A_{3} C_{7}+A_{1}^{9} C_{9}\right)=0 \text {, }
\end{aligned}
$$

If $c_{7} \neq 0$, these equations show that $A_{1}$ and $A_{3}$ are arbitrary, but $A_{4}$ and $A_{5}$ are dependent being given by

$$
A_{4}=A_{1} A_{3} \frac{c_{8}}{c_{7}}, \quad A_{5}=\frac{5 A_{3}^{2}}{A_{1}}+A_{1}^{2} A_{3} \frac{c_{9}}{c_{7}} .
$$

Substituting (27) into the second of equations (26), we obtain the follow- 
ing transformation formulas between $\left(c_{7}, c_{8}, c_{9}, c_{11}\right)$ and $\left(C_{7}, C_{8}, C_{9}, C_{11}\right)$ :

$$
\begin{aligned}
C_{7} & =\beta_{10} A_{1}^{7} c_{7}, \quad C_{8}=\beta_{10} A_{1}^{8} c_{8}, \\
C_{9} & =\beta_{10}\left[A_{1}^{9} c_{9}+7 A_{1}^{6} A_{3} C_{7}\right], \\
C_{11} & =\beta_{10}\left[c_{11} A_{1}^{11}+9 A_{1}^{8} A_{3} c_{9}+8 A_{1}^{7} A_{4} c_{8}+\left(7 A_{1}^{6} A_{5}+21 A_{1}^{5} A_{3}^{2}\right) c_{7}\right] .
\end{aligned}
$$

THEOREM 8. The species $(5,7)$ possesses the absolute invariant

$$
\frac{c_{7}\left(c_{7}^{2} c_{11}-8 c_{7} c_{9}^{2}-8 c_{8}^{2} c_{9}\right)}{c_{8}^{4}} .
$$

The order is 11 , weight is 32 , and degree is 4 .

9. Discussion of the spaces $(5,8)$. The material for our transformed element will be given by the equations of the preceding section if we assume $c_{7}=0$ but $c_{8} \neq 0$.

Since $c_{8} \neq 0$, we find by equations (28) that $A_{1}$ and $A_{4}$ are arbitrary but $A_{3}$ and $A_{5}$ are dependent being given by

$$
A_{3}=0, \quad A_{5}=A_{1} A_{4} \frac{c_{9}}{c_{8}} .
$$

By (30), we see that for this case the transformation formulas between $\left(c_{8}, c_{9}, c_{11}, c_{12}\right)$ and $\left(C_{8}, C_{9}, C_{11}, C_{12}\right)$ are

$$
\begin{aligned}
C_{8} & =\beta_{10} A_{1}^{8} C_{8}, \quad C_{9}=\beta_{10} A_{1}^{9} c_{9}, \\
C_{11} & =\beta_{10}\left[A_{1}^{11} c_{11}+8 A_{1}^{7} A_{4} c_{8}\right], \\
C_{12} & =\beta_{10}\left[A_{1}^{12} c_{12}+9 A_{1}^{8} A_{4} c_{9}+8 A_{1}^{7} A_{5} C_{8}\right] .
\end{aligned}
$$

THEOREM 9. The species $(5,8)$ possesses the absolute invariant

$$
\frac{c_{8}^{2}\left(8 c_{8} c_{12}-9 c_{9} c_{11}\right)}{c_{9}^{4}} .
$$

The order is 12 , weight is 36 , and degree is 4 .

10. Discussion of the species $(4,5)$. In the following, we shall show that this species only possesses a relative differential invariant. This species therefore can be separated into two distinct sets, the elements of any one set being equivalent.

Part of the work for finding the coefficients of the transformed element is the same as that performed for the species $(p, q=p+1)$ for $p>4(\S 6)$. As a matter of fact the first three of equations (21) are valid. From these it follows that the species $(4,5)$ possesses $\left(10 c_{5} c_{7}-11 c_{6}^{2}\right)^{2} / c_{5}^{5}$ as a relative differential invariant. 
In the following, we shall prove that there are no more relative differential invariants. This is done by reducing our species $(4,5)$ to the canonical form.

First let us note that the transformation

$$
X=x+\frac{4 c_{6}}{5 c_{5}^{2}} y, \quad Y=y
$$

will convert our element $(4)$ of species $(4,5)$ into an element whose equation is of the form

$$
y=d_{5} x^{5 / 4}+d_{7} x^{7 / 4}+d_{8} x^{8 / 4}+\cdots,
$$

where the $d$ 's are certain functions of the $c$ 's. Our relative differential invariant for this element is $100 d_{7}^{2} / d_{5}^{3}$ (since $d_{5} \neq 0$ ). Thus we have to discuss this element according as $d_{7} \neq 0$ or $d_{7}=0$.

First let us consider our element (36) for which $d_{7} \neq 0$. For this case, we shall demonstrate the (formal) existence of a transformation which is the $\mathrm{in}$ verse of a transformation of the form

$$
\begin{aligned}
X= & \alpha_{01} x+\alpha_{02} x^{2}+\alpha_{03} x^{3}+\cdots, \\
Y= & \left(\beta_{02} x^{2}+\beta_{03} x^{3}+\beta_{04} x^{4}+\cdots\right)+y\left(\beta_{10}+\beta_{11} x+\beta_{12} x^{2}+\beta_{13} x^{3}+\cdots\right) \\
& +y^{2}\left(\beta_{20}+\beta_{21} x+\beta_{22} x^{2}+\beta_{23} x^{3}+\cdots\right),
\end{aligned}
$$

which will carry our element into the canonical form $y=x^{5 / 4}+x^{7 / 4}$. Therefore it must be shown that the $\alpha_{0 j}$ and the $\beta_{i j}(i=0,1,2)$ can be determined so that our transformation carries the canonical form into the element (36).

Now if we perform this transformation on the canonical form $y=x^{5 / 4}+x^{7 / 4}$, the parametric form of our transformed element is

$$
\begin{aligned}
X= & \alpha_{01} t^{4}+\alpha_{02} t^{8}+\alpha_{03} t^{12}+\cdots, \\
Y= & {\left[\beta_{02} t^{8}+\left(\beta_{03}+2 \beta_{20}\right) t^{12}+\left(\beta_{04}+2 \beta_{21}\right) t^{16}+\cdots\right] } \\
& +\left[\beta_{20} t^{10}+\left(\beta_{21}+\beta_{20}\right) t^{14}+\left(\beta_{22}+\beta_{21}\right) t^{18}+\cdots\right] \\
& +\left[\beta_{10}\left(t^{5}+t^{7}\right)+\beta_{11}\left(t^{9}+t^{11}\right)+\beta_{12}\left(t^{13}+t^{15}\right)+\cdots\right] .
\end{aligned}
$$

The first of these equations defines $t$ as an integral power series in $T$ if we let $X=T^{4}$. This power series must be of the form $t=A_{1} T+A_{5} T^{5}+A_{9} T^{9}+\cdots$ where $A_{1} \neq 0$. If we let $A_{1}^{1 / 4}$ denote a definite fourth root of $A_{1}$, it may be established that there exists a one-to-one correspondence beiween $\left(\alpha_{01}, \alpha_{02}, \cdots, \alpha_{0 n}, \cdots\right)$ and $\left(A_{1}, A_{5}, \cdots, A_{4 n-3}, \cdots\right)$. Therefore we may replace the unknown $\alpha$ 's by the unknown $A$ 's.

In the second of the preceding equations, let us replace the coefficient of $t^{4 n+4}$ by $B_{4 n+4}$, the coefficient of $t^{4 n+6}$ by $B_{4 n+6}$, and the coefficient of $t^{4 n+1}$ by $B_{4 n+1}$ where $n=1,2,3, \cdots$. Then obviously there exists a one-to-one correspondence between the $\beta_{i j}$ and the $B_{k}$. Thus we may substitute the unknown $B$ 's for the unknown $\beta$ 's. 
By the preceding remarks, our problem then is to determine the $A$ 's and the $B$ 's so that the equations

$$
\begin{aligned}
X= & T^{4}, \quad t=A_{1} T+A_{5} T^{5}+A_{9} T^{9}+\cdots \\
Y= & {\left[B_{8} t^{8}+B_{12} t^{12}+B_{16} t^{16}+\cdots\right]+\left[B_{10} t^{10}+B_{14} t^{14}+B_{18} t^{18}+\cdots\right] } \\
& +\left[B_{5}\left(t^{5}+t^{7}\right)+B_{9}\left(t^{9}+t^{11}\right)+B_{13}\left(t^{13}+t^{15}\right)+\cdots\right],
\end{aligned}
$$

shall represent the element (36) upon eliminating the parameters $t$ and $T$.

Performing this elimination, we find our equivalence equations to be

$$
\begin{aligned}
& B_{8} A_{1}^{8}=d_{8}, \quad B_{12} A_{1}^{12}+8 B_{8} A_{1}^{7} A_{6}=d_{12}, \\
& B_{4 n+4} A_{1}^{4 n+4}+\left(\text { lower } B_{4 k}\right)=d_{4 n+4} \text {, } \\
& B_{10} A_{1}^{10}=d_{10}, \quad B_{14} A_{1}^{14}+10 B_{10} A_{1}^{9} A_{5}=d_{14}, \\
& B_{4 n+6} A_{1}^{4 n+6}+\left(\text { lower } B_{4 k+6}\right)=d_{4 n+6}, \\
& B_{5} A_{1}^{5}=d_{5}, \quad 5 A_{1}^{4} A_{5} B_{5}+B_{9} A_{1}^{9}=d_{9}, \\
& B_{5} A_{1}^{7}=d_{7}, \quad 7 A_{1}^{6} A_{5} B_{5}+B_{9} A_{1}^{11}=d_{11} \text {, } \\
& 5 A_{1}^{4} A_{4 n-3} B_{5}+B_{4 n+1} A_{1}^{4 n+1}+\left(\text { lower } A_{4 k-3} \text { and } B_{4 k+1}\right)=d_{4 n-3} \text {, } \\
& 7 A_{1}^{6} A_{4 n-3} \dot{B}_{5}+B_{4 n+1} A_{1}^{4 n+3}+\left(\text { lower } A_{4 k-3} \text { and } B_{4 k+1}\right)=d_{4 n-1} \text {, }
\end{aligned}
$$

The first pair of the equations (40.3) shows that $A_{1} \neq 0$ and $B_{5} \neq 0$ are uniquely determined. The second pair demonstrates that $A_{5}$ and $B_{9}$ are uniquely determined since the determinant of the coefficients $-2 A_{1}^{15} B_{5} \neq 0$. The $n$th pair proves that $A_{4 n-3}$ and $B_{4 n+1}$ are uniquely evaluated since the determinant of the coefficients $-2 A_{1}^{4 n+7} B_{5} \neq 0$. Thus by induction, all the $A_{4 n-3}$ and the $B_{4 n+1}$ are uniquely found.

The equations (40.1) and (40.2) uniquely evaluate the $B_{4 n+4}$ and the $B_{4 n+6}$, respectively. Hence we are able to find unique values for all the $A$ 's and the $B$ 's and therefore (39) actually represents our element (36). Thus the species $(4,5)$ for which the relative differential invariant $\left(10 c_{5} c_{7}-11 c_{6}^{2}\right)^{2} / c_{5}^{5} \neq 0$ possesses no additional invariants. The canonical form is $y=x^{5 / 4}+x^{7 / 4}$. 
Finally let us consider the case for which our relative differential invariant is zero. Then for our element (36) we must have $d_{7}=0$. The inverse of the transformation

$$
X=x, \quad Y=y+d_{8} x^{2}-\frac{d^{9}}{d_{5}} x y-\frac{d_{10}}{d_{5}^{2}} y^{2},
$$

will then carry our element (36) into one of the form

$$
y=e_{5} x^{5 / 4}+e_{11} x^{11 / 4}+e_{12} x^{12 / 4}+\cdots,
$$

where the $e$ 's are certain functions of the $d$ 's and hence it follows that they are functions of the $c$ 's.

Next the transformation which is the inverse of the correspondence

$$
X=x+\frac{4 e_{11}}{5 e_{5}^{3}} y, \quad Y=y,
$$

will carry our element (42) into an element of the form

$$
y=f_{5} x^{5 / 4}+f_{12} x^{12 / 4}+f_{13} x^{13 / 4}+\cdots,
$$

where the $f$ 's are certain functions of the $e$ 's and hence of the $c$ 's.

Finally the inverse of the transformation

$$
\begin{aligned}
X=x, \quad Y= & \left(f_{12} x^{3}+f_{16} x^{4}+f_{20} x^{5}+\cdots\right) \\
& +y\left(f_{5}+f_{13} x^{2}+f_{17} x^{3}+f_{21} x^{4}+\cdots\right) \\
& +y^{2}\left(f_{14}+f_{18} x^{2}+f_{22} x^{3}+\cdots\right) \\
& +y^{3}\left(f_{15}+f_{19} x+f_{23} x^{2}+\cdots\right)
\end{aligned}
$$

will carry our element $(44)$ into the element $y=x^{5 / 4}$. Thus the species $(4,5)$ for which the relative differential invariant $\left(10 c_{5} c_{7}-11 c_{6}^{2}\right)^{2} / c_{5}^{2}=0$ possesses no additional invariants. The canonical form is $y=x^{5 / 4}$.

Therefore we have proved the following result.

Theorem 10. The species $(4,5)$ possesses only the relative differential invariant

$$
\frac{\left(10 c_{5} c_{7}-11 c_{6}^{2}\right)^{2}}{c_{5}^{5}}
$$

and no other differential or arithmetic invariants. Thus our species $(4,5)$ may be separated into two distinct sets according as this relative differential invariant is not or is zero. The canonical forms of these two distinct sets are $y=x^{5 / 4}+x^{7 / 4}$, and $y=x^{5 / 4}$, respectively.

11. Discussion of the species $(4,7)$. For this case, we see that the parametric equations (5) for our new element assume the form 


$$
\begin{aligned}
X= & \alpha_{01} t^{4}+\alpha_{10} c_{7} t^{7}+\left(\alpha_{02}+\alpha_{10} c_{8}\right) t^{8}+\alpha_{10}\left(c_{9} t^{9}+c_{10} t^{10}\right)+\cdots, \\
Y= & \beta_{10} c_{7} t^{7}+\left(\beta_{02}+\beta_{10} c_{8}\right) t^{8}+\beta_{10}\left(c_{9} t^{9}+c_{10} t^{10}\right) \\
& +\left(\beta_{11} c_{7}+\beta_{10} c_{11}\right) t^{11}+\left(\beta_{03}+\beta_{10} c_{12}+\beta_{11} c_{8}\right) t^{12} \\
& +\left(\beta_{11} c_{9}+\beta_{10} c_{13}\right) t^{13}+\cdots .
\end{aligned}
$$

Let $X=T^{4}$. The first of these equations defines $t$ as an integral power series in $T$. This power series must be of the form

$$
t=A_{1} T+A_{4} T^{4}+A_{5} T^{5}+A_{6} T^{6}+A_{7} T^{7}+\cdots, \quad A_{1} \neq 0 .
$$

Upon replacing $t$ by this value in the first of equations (47), we obtain the following system of equations

$$
\begin{aligned}
\alpha_{01} A_{1}^{4}=1, \quad 4 \alpha_{01} A_{1}^{3} A_{4}+\alpha_{10} A_{1}^{7} c_{7} & =0, \\
4 \alpha_{01} A_{1}^{3} A_{5}+\left(\alpha_{02}+\alpha_{10} c_{8}\right) A_{1}^{8} & =0, \\
4 \alpha_{01} A_{1}^{3} A_{6}+\alpha_{10} A_{1}^{9} c_{9} & =0, \\
\alpha_{01}\left(4 A_{1}^{3} A_{7}+6 A_{1}^{2} A_{4}^{2}\right)+\alpha_{10}\left(7 A_{1}^{6} A_{4} c_{7}+A_{1}^{10} c_{10}\right) & =0,
\end{aligned}
$$

By these equations, we find, since $c_{7} \neq 0$, that $A_{1}, A_{4}$, and $A_{5}$ are arbitrary. But the values of $A_{6}$ and $A_{7}$ are dependent, being given by

$$
\begin{aligned}
& A_{6}=\frac{c_{9}}{c_{7}} A_{1}^{2} A_{4}, \\
& A_{7}=\frac{11 A_{4}^{2}}{2 A_{1}}+\frac{c_{10}}{c_{7}} A_{1}^{3} A_{4} .
\end{aligned}
$$

Upon substituting (48) into the second of equations (47), we obtain the following transformation formulas between $\left(c_{7}, c_{8}, c_{9}, c_{10}, c_{11}, c_{12}, c_{13}\right)$ and $\left(C_{7}, C_{8}, C_{9}, C_{10}, C_{11}, C_{12}, C_{13}\right)$ :

$$
\begin{aligned}
C_{7}= & \beta_{10} A_{1}^{7} C_{7}, \quad C_{8}=\beta_{10} A_{1}^{8} c_{8}+\beta_{02} A_{1}^{8}, \\
C_{9}= & \beta_{10} A_{1}^{9} C_{9}, \quad C_{10}=\beta_{10}\left(A_{1}^{10} c_{10}+7 A_{1}^{6} A_{4} C_{7}\right), \\
C_{11}= & 8 \beta_{02} A_{1}^{7} A_{4}+\beta_{11} A_{1}^{11} c_{7}+\beta_{10}\left(c_{11} A_{1}^{11}+8 A_{1}^{7} A_{4} C_{8}+7 A_{1}^{6} A_{5} C_{7}\right), \\
C_{12}= & 8 \beta_{02} A_{1}^{7} A_{5}+\beta_{03} A_{1}^{12}+\beta_{11} A_{1}^{12} c_{8} \\
& +\beta_{10}\left(A_{1}^{12} c_{12}+9 A_{1}^{8} A_{4} C_{9}+8 A_{1}^{7} A_{5} C_{8}+7 A_{1}^{6} A_{6} C_{7}\right), \\
C_{13}= & 8 \beta_{02} A_{1}^{7} A_{6}+\beta_{11} A_{1}^{13} c_{9} \\
& +\beta_{10}\left[A_{1}^{13} c_{13}+10 A_{1}^{9} A_{4} c_{10}+9 A_{1}^{3} A_{5} C_{9}+8 A_{1}^{7} A_{6} C_{8}\right. \\
& \left.\quad+c_{7}\left(7 A_{1}^{6} A_{7}+21 A_{1}^{5} A_{4}^{2}\right)\right] .
\end{aligned}
$$


By these equations, we discover that the species $(4,7)$ possesses $c_{9}^{3} / c_{7}^{4}$ as a relative differential invariant. Moreover if this relative invariant is zero $\left(c_{9}=0\right)$, then the species $(4,7)$ will possess $\left(14 c_{7} c_{13}-17 c_{10}^{2}\right) / c_{7}^{3}$ as a relative differential invariant. In the remainder of this section, we shall demonstrate that there are no additional invariants. This is accomplished by reducing our element of species $(4,7)$ to the canonical form.

In the first place, it is observed that the transformation

$$
X=x+\frac{4 c_{10}}{7 c_{7}^{2}} y, \quad Y=y,
$$

will carry our element $(4)$ of species $(4,7)$ into an element of the form

$$
y=d_{7} x^{7 / 4}+d_{8} x^{8 / 4}+d_{9} x^{9 / 4}+d_{11} x^{11 / 4}+\cdots,
$$

where the $d$ 's are certain functions of the $c$ 's. Our first relative differential invariant for this element is $d_{9}^{3} / d_{7}^{4}$. Thus we have to discuss this element according as $d_{9} \neq 0$ or $d_{9}=0$.

Let us now consider the case where $d_{9} \neq 0$. By a very similar argument to the one given near the beginning of $\S 10$, it is very easy to demonstrate the (formal) existence of a unique transformation which is the inverse of a correspondence of the form (37) which will carry out element (53) into the canonical form $y=x^{7 / 4}+x^{9 / 4}$. Therefore the species $(4,7)$ for which the relative differential invariant $c_{9}^{3} / c_{7}^{4} \neq 0$ possesses no additional invariants. The normal form is $y=x^{7 / 4}+x^{8 / 4}$.

Next let us consider the case where $d_{9}=0$. For our element (53), our second relative differential invariant is $14 d_{13} / d_{7}^{2}$. We next have to consider this element according as $d_{13} \neq 0$ or $d_{13}=0$.

We observe that in any case (whether $d_{13} \neq 0$ or $d_{13}=0$ ) that the transformation

$$
X=x+\frac{4 d_{11}}{7 d_{7}} x^{2}, \quad Y=y,
$$

will carry our element (53) with $d_{9}=0$ into an element of the form

$$
y=e_{7} x^{7 / 4}+e_{8} x^{8 / 4}+e_{12} x^{12 / 4}+e_{13} x^{13 / 4}+\cdots .
$$

It remains to discuss this element according as $e_{13} \neq 0$ or $e_{13}=0$.

Let us now consider the case where $e_{13} \neq 0$. By an argument very similar to the one given near the beginning of $\$ 10$, it is easy to prove the (formal) existence of a unique transformation which is the inverse of a correspondence of the form (37) which will carry our element (55) into the canonical form $y=x^{7 / 4}+x^{13 / 4}$. Therefore the species $(4,7)$ for which $c_{9}=0$ and the relative differential invariant $\left(14 c_{7} c_{13}-17 c_{10}^{2}\right) / c_{7}^{3} \neq 0$ possesses no other invariants. The canonical form is $y=x^{7 / 4}+x^{13 / 4}$. 
Finally it remains to consider the case where $e_{13}=0$. It is observed that the transformation

$$
X=x+\frac{4 c_{17}}{7 c_{7}^{3}} y, \quad Y=y,
$$

will carry our element (55) into one of the form

$$
\begin{aligned}
y= & f_{7} x^{7 / 4}+f_{8} x^{8 / 4}+f_{12} x^{12 / 4}+f_{14} x^{14 / 4}+f_{15} x^{15 / 4}+f_{16} x^{16 / 4} \\
& +f_{18} x^{18 / 4}+\cdots,
\end{aligned}
$$

where the $f$ 's are certain functions of the $e$ 's and therefore of the $c$ 's.

The transformation whose inverse is

$$
\begin{aligned}
X=x, \quad Y= & \left(f_{8} x^{2}+f_{12} x^{3}+f_{16} x^{4}+\cdots\right) \\
& +y\left(f_{7}+f_{15} x^{2}+f_{19} x^{3}+\cdots\right) \\
& +y^{2}\left(f_{14}+f_{18} x+f_{22} x^{2}+\cdots\right) \\
& +y^{3}\left(f_{21}+f_{25} x+f_{29} x^{2}+\cdots\right),
\end{aligned}
$$

will carry our element (57) into the normal form $y=x^{7 / 4}$. Therefore the species $(4,7)$ for which $c_{9}=0$ and $14 c_{7} c_{13}-17 c_{10}^{2}=0$ possesses no additional invariants. The canonical form is $y=x^{7 / 4}$.

We thus may make the following statement.

Theorem 11. The species $(4,7)$ possesses the relative differential invariant

$$
c_{9}^{3} / c_{7}^{4}
$$

If this relative differential invariant is zero, then that set of those elements of this species for which $c_{9}=0$ possesses the additional relative differential invariant

$$
\frac{14 c_{7} c_{13}-17 c_{10}^{2}}{c_{7}^{3}} .
$$

The species $(4,7)$ does not possess any more invariants. Therefore our species $(4,7)$ has been divided into the following three distinct sets:

(A) Those for which $c_{9} \neq 0$. The canonical form is $y=x^{7 / 4}+x^{9 / 4}$.

(B) Those for which $c_{9}=0$ but $14 c_{7} c_{13}-17 c_{10}^{2} \neq 0$. The canonical form is $y=x^{7 / 4}+x^{13 / 4}$.

(C) Those for which $c_{9}=0$ and $14 c_{7} c_{13}-17 c_{10}^{2}=0$. The canonical form is $y=x^{7 / 4}$.

12. Discussion of the species $(4,6)$. It is clear that any curve of this species must be of the form

$$
y=c_{6} x^{6 / 4}+c_{8} x^{8 / 4}+\cdots+c_{2 \rho} x^{2 \rho / 4}+c_{Q} x^{Q / 4}+\cdots,
$$


where not only $c_{\theta} \neq 0$ but also $c_{Q} \neq 0$ where $Q=2 \rho+1 \geqq 7$ denotes the first odd power of the fourth root of $x$ which appears in the series expansion for $y$. For otherwise our element would simply be an element of the species $(2,3)$, which shall be discussed at the end of our paper.

Upon substituting this into the equations (10) defining the group $G^{\prime}$, we find the parametric equations of the transformed element to be

$$
\begin{aligned}
& X=\gamma_{4} t^{4}+\alpha_{10} c_{6} t^{6}+\gamma_{8} t^{8}+\gamma_{10} t^{10}+\cdots+\gamma_{2 \rho} t^{2 \rho}+\alpha_{10} c_{2 \rho+1} t^{2 \rho+1}+\cdots, \\
& Y=\beta_{10} c_{6} t^{6}+\delta_{8} t^{8}+\delta_{10} t^{10}+\cdots+\delta_{2 \rho} t^{2 \rho}+\beta_{10} c_{2 \rho+1} t^{2 \rho+1}+\cdots,
\end{aligned}
$$

where $\gamma_{4}$ and $\beta_{10}$ are any two nonzero numbers and the remaining $\gamma$ and $\delta$ are arbitrary numbers.

Let $X=T^{4}$. The first of the preceding equations defines $t$ as an integral power series in $T$. This power series must be of the form

$$
t=A_{1} T+A_{3} T^{3}+A_{5} T^{5}+\cdots+A_{2 \rho-3} T^{2 \rho-3}+A_{2 \rho-2} T^{2 \rho-2}+\cdots .
$$

Until the $(2 \rho-3)$ term only odd powers appear. Thereafter the exponents of $T$ increase by one (as far as we know).

Substituting this into the first of equations (62), we discover that all the $A_{1} \neq 0, A_{3}, A_{5}, \cdots, A_{2 \rho-3}$ are arbitrary but that we have $A_{2 \rho-2}=c_{2 \rho+1} A_{1}^{2 \rho-5} A_{3} / c_{6}$.

Finally replacing $t$ by this series into the second of equations (62), we obtain the following transformation formulas between $\left(c_{6}, c_{2 \rho+1}, c_{2 \rho+3}\right)$ and $\left(C_{6}, C_{2 \rho+1}, C_{2 \rho+3}\right)$

$$
\begin{aligned}
C_{6} & =\beta_{10} A_{1}^{6} c_{6}, \quad C_{2 \rho+1}=\beta_{10} A_{1}^{2 \rho+1} c_{2 \rho+1}, \\
C_{2 \rho+3} & =\beta_{10}\left[(2 \rho+7) A_{1}^{-2 \rho} A_{3} c_{2 \rho+1}+A_{1}^{2 \rho+3} c_{2 \rho+3}\right] .
\end{aligned}
$$

From these results we immediately deduce that the integer $Q=2 \rho+1 \geqq 7$ is an arithmetic invariant and $c_{Q}^{5} / c_{\theta}^{Q-1}$ is a relative differential invariant. In the remainder of this section, we shall prove that there are no more invariants.

First the transformation

$$
X=x+\frac{4 c_{2 \rho+3}}{(2 \rho+7) c_{6} c_{2 \rho+1}} y, \quad Y=y
$$

will convert our arc (61) into an element of the form

$$
\begin{aligned}
y= & d_{6} x^{6 / 4}+d_{8} x^{8 / 4}+\cdots+d_{2 \rho} x^{2 \rho / 4}+d_{2 \rho+1} x^{(2 \rho+1) / 4} \\
& +d_{2 \rho+2} x^{(2 \rho+2) / 4}+d_{2 \rho+4} x^{(2 \rho+4) / 4}+\cdots,
\end{aligned}
$$

where the $d$ 's are certain functions of the $c$ 's. We note that our transformation has eliminated the $(2 \rho+3)$ rd power of the fourth root of $x$.

Now we shall demonstrate the existence of a transformation which is the inverse of a correspondence of the form (37), which will carry our element (66) 
into the canonical form $y=x^{3 / 2}+x^{Q / 4}$. Thus the $\alpha_{0 j}$ and the $\beta_{i j}(i=0,1,2)$ must be determined so that this correspondence (37) carries the canonical form into our element (66).

Now if we apply our transformation (37) to the canonical form $y=x^{6 / 4}$ $+x^{(2 \rho+1) / 4}$, we find as the parametric form of the transformed element

$$
\begin{aligned}
X= & \alpha_{01} t^{4}+\alpha_{02} t^{8}+\alpha_{03} t^{12}+\cdots, \\
Y= & {\left[\beta_{02} t^{8}+\left(\beta_{03}+\beta_{20}\right) t^{12}+\cdots+\left(\beta_{0, n+2}+\beta_{2, n-1}\right) t^{4 n+8}+\cdots\right] } \\
& +\left[\beta_{10} t^{2 \rho+1}+\beta_{11} t^{2 \rho+5}+\beta_{12} t^{2 \rho+9}+\cdots+\beta_{1, \rho-2} t^{6 \rho-7}\right. \\
& \left.\quad+\beta_{1, \rho-1} t^{6 \rho-3}+\beta_{1, \rho} t^{6 \rho+1}+\cdots+\beta_{1, \rho-2+n} t^{6 \rho-7+4 n}+\cdots\right] \\
& +\left[2 \beta_{20} t^{2 \rho+7}+2 \beta_{21} t^{2 \rho+11}+\cdots+2 \beta_{2, \rho-2} t^{6 \rho-1}\right. \\
& \left.+2 \beta_{2, \rho-1} t^{6 \rho+3}+\cdots+2 \beta_{2, \rho-2+n} t^{6 \rho-1+4 n}+\cdots\right] \\
& +\left[\beta_{10} t^{6}+\beta_{11} t^{10}+\cdots+\beta_{1, \rho-2} t^{4 \rho-2}+\left(\beta_{1, \rho-1}+\beta_{20}\right) t^{4 \rho+2}\right. \\
& \left.+\left(\beta_{1, \rho}+\beta_{21}\right) t^{4 \rho+6}+\cdots+\left(\beta_{1, \rho-2+n}+\beta_{2, n-1}\right) t^{4 \rho-2+4 n}+\cdots\right] .
\end{aligned}
$$

In the second of the preceding equations, let us replace the coefficients of $t^{6}, t^{10}, t^{14}, \cdots, t^{4 \rho-2}$ by $B_{6}, B_{10}, B_{14}, \cdots, B_{4 \rho-2}$, respectively, the coefficient of $t^{6 \rho-7+4 n}$ by $B_{6-7+4 n}$, the coefficient of $t^{2 \rho+3+4 n}$ by $B_{2 \rho+3+4 n}$, and the coefficient of $t^{4 n+4}$ by $B_{4 n+4}$ for $n=1,2,3, \cdots$. There obviously exists a one-to-one correspondence between the $\beta_{i j}$ and the $B_{k}$.

Therefore our problem is to determine the $A$ 's and the $B$ 's so that the equations

$$
\begin{aligned}
X= & T^{4}, \quad t=A_{1} T+A_{5} T^{5}+A_{9} T^{9}+\cdots \\
Y= & {\left[B_{8} t^{8}+B_{12} t^{12}+\cdots+B_{4 n+4} t^{4 n+4}+\cdots\right] } \\
& +\left[B_{6}\left(t^{6}+t^{2 \rho+1}\right)+B_{10}\left(t^{10}+t^{2 \rho+5}\right)+\cdots+B_{4 \rho-2}\left(t^{4 \rho-2}+t^{6 \rho-7}\right)\right] \\
& +\left[B_{6 \rho-3} t^{6 \rho-3}+B_{6 \rho+1} t^{6 \rho+1}+\cdots+B_{6 \rho-7+4 n} t^{6 \rho-7+4 n}+\cdots\right] \\
& +\left[2 B_{2 \rho+7} t^{2 \rho+7}+2 B_{2 \rho+11} t^{2 \rho+11}+\cdots+2 B_{6 \rho-1} t^{6 \rho-1}\right. \\
& \left.\quad+2 B_{6 \rho+3} t^{6 \rho+3}+2 B_{6 \rho+7} t^{6 \rho+7}+\cdots+2 B_{6 \rho-1+4 n} l^{6 \rho-1+4 n}+\cdots\right] \\
& +\left[\left(B_{6 \rho-3}+B_{2 \rho+7}\right) t^{4 \rho+2}+\left(B_{6 \rho+1}+B_{2 \rho+11} t^{4 \rho+6}+\cdots\right.\right. \\
& \left.+\left(B_{6 \rho-7+4 n}+B_{2 \rho+3+4 n}\right) t^{4 \rho-2+4 n}+\cdots\right]
\end{aligned}
$$

shall represent the element (66) upon elimination of the parameters $t$ and $T$.

The elimination yields the following equivalence equations:

$$
B_{8} A_{1}^{8}=d_{8}, \quad B_{12} A_{1}^{12}+8 B_{8} A_{1}^{\bar{j}} A_{5}=d_{12},
$$

$$
B_{4 n+4} \Lambda_{1}^{4 n+4}+\left(\text { lower } B_{4 k}\right)=d_{4 n+4},
$$


$(69.2)$

$$
\begin{aligned}
B_{6} A_{1}^{6} & =d_{6}, & 6 A_{1}^{5} A_{5} B_{6}+B_{10} A_{1}^{10} & =d_{10}, \\
B_{6} A_{1}^{2 \rho+1} & =d_{2 \rho+1}, & (2 \rho+1) A_{1}^{2 \rho} A_{5} B_{6}+B_{10} A_{1}^{2 \rho+5} & =d_{2 \rho+5},
\end{aligned}
$$

$$
\begin{aligned}
6 A_{1}^{5} A_{4 \rho-7} B_{6}+B_{4 \rho-2} A_{1}^{4 \rho-2}+\left(\text { lower } A_{4 k-3} \text { and } B_{4 k-2}\right) & =d_{4 \rho+2}, \\
(2 \rho+1) A_{1}^{2 \rho} A_{4 \rho-7} B_{6}+B_{4 \rho-2} A_{1}^{6 \rho-2}+\left(\text { lower } A_{4 k-3} \text { and } B_{4 k-2}\right) & =d_{6 \rho-7} ; \\
(2 \rho+1) A_{1}^{2 \rho} A_{4 \rho-3} B_{6}+B_{6 \rho-3} A_{1}^{6 \rho-3}+\left(\text { lower } A_{4 k-3} \text { and } B_{2 \rho-3+4 k}\right) & =d_{6 \rho-3}, \\
2 B_{2 \rho+7} A_{1}^{7} & =d_{2 \rho+7}, \\
6 A_{1}^{5} A_{4 \rho-3} B_{6}+\left(B_{6 \rho-3}+B_{2 \rho+7}\right) A_{1}^{4 \rho+2}+\left(\text { lower } A_{4 k-3} \text { and } B_{4 k+2}\right) & =d_{4 \rho+2},
\end{aligned}
$$

$$
\begin{aligned}
(2 \rho+1) A_{1}^{2 \rho} A_{4 \rho-7+4 n} B_{6}+B_{6 \rho-7+4 n} A_{1}^{6 \rho-7+4 n}+\left(\text { lower } A_{4 k-3}\right. \text { and } & \left.B_{2 \rho-3+4 k}\right) \\
& =d_{6 \rho-i+4 n}, \\
2 B_{2 \rho+3+4 n} A_{1}^{2 \rho+3+4 n}+\left(\text { lower } A_{4 k-3} \text { and } B_{2 \rho+3+4 k}\right) & =d_{2 \rho+3+4 n}, \\
6 A_{1}^{5} A_{4 \rho-7+4 n} B_{6}+\left(B_{6 \rho-7+4 n}+B_{2 \rho+3+4 n}\right) A_{1}^{4 \rho-6+4 n}+\cdots & =d_{4 \rho-6+4 n},
\end{aligned}
$$

The first pair of equations (69.2) furnishes unique values for $A_{1} \neq 0$ and $B_{6} \neq 0$. The second pair yields unique values for $A_{5}$ and $B_{10}$ since the determinant of the coefficients $(-2 \rho+5) A_{1}^{2 \rho+10} B_{6} \neq 0$. The last pair give unique evaluations for $A_{4 \rho-7}$ and $B_{4 \rho-2}$ since the determinant of the coefficients $(-2 \rho+5) A_{1}^{6 \rho-2} B_{6} \neq 0$. Thus the equations (69.2) determine the values of $A_{1} \neq 0, A_{5}, \cdots, A_{4 \rho-7}$; and $B_{6} \neq 0, B_{10}, \cdots, B_{4 \rho-2}$ uniquely.

The first triplet of the equations (69.3) determines unique values for $A_{4 \rho-3}$, $B_{6 \rho-3}$, and $B_{2 \rho+7}$, since the determinant of the coefficients $2(2 \rho-5) A_{1}^{8 \rho+9} B_{6} \neq 0$. The $n$th triplet furnishes unique evaluations for $A_{4 \rho-7+4 n}, B_{6 \rho-7+4 n}$, and $B_{2 \rho+3+4 n}$ since the determinant of the coefficients $2(2 \rho-5) A_{1}^{8 \rho+5+4 n} B_{6} \neq 0$. The equations (69.3) therefore give unique values for $A_{4 \rho-3}, A_{4 \rho+1}, \cdots, A_{4 \rho-7+4 n}, \cdots$; $B_{6 \rho-3}, B_{6 \rho+1}, \cdots, B_{6 \rho-7+4 n}, \cdots$; and $B_{2 \rho+7}, B_{2 \rho+11}, \cdots, B_{2 \rho+3+4 n}, \cdots$.

Finally the equations (69.1) obviously determine the values of $B_{8}$, $B_{12}, \cdots, B_{4 n+4}, \cdots$ uniquely. Therefore this proves that the canonical form of the species $(4,6)$ is $y=x^{3 / 2}+x^{(2 \rho+1) / 4}$ where $\rho \geqq 3$.

We are now in a position to state the following result.

THEOREM 12. The species $(4,6)$ possesses the odd integer $Q \geqq 7$, the first odd power of the fourth root of $x$ which appears in the series expansion for $y$, as an arithmetic invariant, and the expression

$$
\frac{c_{Q}^{5}}{c_{6}^{Q-1}}
$$


as a relative differential invariant. There are no further invariants. The canonical form is $y=x^{3 / 2}+x^{Q / 4}$.

13. Discussion of the species $(3, q)$. Any element of this species may be written in the form

$$
y=c_{q} x^{q / 3}+c_{q_{1}} x^{q_{1} / 3}+c_{q_{2}} x^{q_{2} / 3}+\cdots+c_{q_{n-1}} x^{q_{n-1} / 3}+c_{Q} x^{Q / 3}+\cdots,
$$

where $q=3 r+s(0<s<3)$ and the integers $q<q_{1}<q_{2}<\ldots<q_{n-1}<Q$ are such that $q+q_{i} \neq \equiv \bmod 3(i=1,2, \cdots, n-1)$ but $q+Q \equiv 0 \bmod 3$.

The correspondent of this element under any transformation of our group $G^{\prime}$ (equations (12)) is given by the parametric form

$$
\begin{aligned}
X= & \alpha_{01} t^{3}+\alpha_{02} t^{6}+\cdots+\alpha_{0 r} t^{3 r}+\alpha_{10} c_{3 r+8} t^{3 r+s}+\cdots, \\
Y= & {\left[\beta_{0, r+1} t^{3 r+3}+\beta_{0, r+2} t^{3 r+6}+\cdots\right] } \\
& +\beta_{10}\left[c_{q} t^{q}+c_{q_{1}} t^{q_{1}}+c_{q_{2}} t^{q_{2}}+\cdots+c_{q_{n-1}} t^{q_{n-1}}+c_{Q} t^{Q}+\cdots\right] \\
& +\beta_{11}\left[c_{q} t^{q+3}+c_{q_{1}} t^{q_{1}+3}+c_{q_{2}} t^{q_{2}+3}+\cdots\right] \\
& +\cdots
\end{aligned}
$$

By the first of these equations, we find that $t$ is defined as an integral power series in $T$ where $X=T^{3}$. This power series must be of the form

$$
t=A_{1} T+A_{4} T^{4}+A_{7} T^{7}+\cdots+A_{3 r-2} T^{3 r-2}+A_{3 r-1} T^{3 r-1}+\cdots
$$

where the exponents after the last written term increase by one (as far as we know). Substituting this into the first of equations (72), we discover that $\left(A_{1} \neq 0, A_{4}, A_{7}, \cdots, A_{3 r-2}, A_{3 r-1}\right)$ may be taken as arbitrary quantities.

Now let us consider the case where $Q<2 q-3$. In this case, it is found upon substituting (73) into the second of equations (72) that the following transformation formulas exist between $\left(c_{q}, c_{Q}\right)$ and $\left(C_{q}, C_{Q}\right)$ :

$$
C_{q}=\beta_{10} A_{1 c_{q}}^{q}, \quad C_{Q}=\beta_{10} A_{1}^{Q} c_{Q} .
$$

Thus the integer $Q$ is an arithmetic invariant (since $c_{Q} \neq 0$ and hence $C_{Q} \neq 0$ ), and the expression $c_{Q}^{Q-1} / c_{Q}^{Q-1}$ is a relative differential invariant.

Next, by examining the cases $s=1$ and $s=2$ separately it may be shown that there exists a unique transformation which is the inverse of a correspondence of the form

$$
\begin{aligned}
& X=\alpha_{01} x+\alpha_{02} x^{2}+\alpha_{03} x^{3}+\cdots, \\
& Y=\left(\beta_{0, r+1} x^{r+1}+\beta_{0, r+2} x^{r+2}+\cdots\right)+y\left(\beta_{10}+\beta_{11} x+\beta_{12} x^{2}+\cdots\right),
\end{aligned}
$$

which carries our element (71) for which $Q<2 q-3$ into the normal form $y=x^{q / 3}+x^{Q / 3}$. Therefore the canonical form of the species $(3, q)$ for which $Q$ is the first power of the cube root of $x$ in the series expansion for $y$ such that $q+Q \equiv 0 \bmod 3$ and $q<Q<2 q-3$ is $y=x^{q / 3}+x^{Q / 3}$. 
It remains now to examine the case for which $Q \geqq 2 q-3$. First it may easily be verified that there exists a transformation which is the inverse of a correspondence of the form

$$
\begin{aligned}
X=x, \quad Y= & \left(\beta_{r+1,0} x^{r+1}+\beta_{r+2,0} x^{r+2}+\cdots+\beta_{r+n, 0} x^{r+n}\right) \\
& +y\left(1+\beta_{11} x+\beta_{12} x^{2}+\cdots+\beta_{1, r-1} x^{r-1}\right),
\end{aligned}
$$

where $n=r-1$ or $r$ according as $s=1$ or 2 , which carries our element (71) into one of the form

$$
y=d_{q} x^{q / 3}+d_{2 q-3} x^{(2 q-3) / 3}+\cdots,
$$

where the $d$ 's are certain functions of the $c$ 's.

Next the point transformation

$$
X=x+\frac{3 d_{2 q-3}}{q d_{q}^{2}} y, \quad Y=y
$$

carries our element (77) into one of the form

$$
y=e_{q} x^{q / 3}+e_{2 q-2} x^{(2 q-2) / 3}+\cdots .
$$

Finally it may very easily be shown by examining the cases $s=1$ and $s=2$ separately that there exists a transformation which is the inverse of the correspondence $X=x, Y=\psi(x, y)$ which carries our element (79) into the standard form $y=x^{q / 3}$. Therefore the canonical form of the species $(3, q)$ for which $Q$ is the first power of the cube root of $x$ in the series expansion for $y$ such that $q+Q \not \equiv 0 \bmod 3$ and $Q \geqq 2 q-3$ is $y=x^{q / 3}$.

The following statement is thus found.

TheOREM 13. In the species $(3, q)$ let $Q$ be the first power of the cube root of $x$ in the series expansion for $y$ such that $q+Q \equiv 0 \bmod 3$. If $Q$ is such that $q<Q<2 q-3$, then our species possesses $Q$ as an arithmetic invariant and the expression

$$
\frac{c_{Q}^{q-1}}{c_{q}^{Q-1}}
$$

as a relative differential invariant. For this case, there are no further relative invariants, the canonical form being $y=x^{q / 3}+x^{Q / 3}$. On the other hand if $Q \geqq 2 q-3$, there are no invariants and the canonical form is $y=x^{q / 3}$.

14. Discussion of the species $(2, q)$. Obviously the inverse of the transformation

$$
\begin{aligned}
X=x, \quad Y= & \left(c_{2 r+2} x^{r+1}+c_{2 r+4} x^{r+2}+\cdots\right) \\
& +y\left(c_{2 r+1}+c_{2 r+3} x+c_{2 r+5} x^{2}+\cdots\right)
\end{aligned}
$$


carries any element of our species into the element $y=x^{(2 r+1) / 2}$. Hence

Theorem 14. The canonical form of the species $(2, q)$ is $y=x^{q / 2}$. There are no differential invariants. The only arithmetic invariant is $q$.

This completes our classification of analytic elements. This has been based on the type of lowest order differential invariant. (Higher invariants will be discussed elsewhere.) Accordingly our classification leads to one regular type and twelve irregular types. This is more complicated than the conformal classification discussed elsewhere $\left({ }^{1}\right)$. Projective invariants of irregular elements (besides Halphen's regular invariant) exist in great variety.

Columbia University,

NEW YoRK, N. Y.

ILLINOIS INSTISUTE OF TECHNOLOGY,

Chicago, Ill. 